\title{
Overview of homocysteine and folate metabolism. With special references to cardiovascular disease and neural tube defects
}

\author{
Henk J. Blom • Yvo Smulders
}

Received: 24 February 2010 /Revised: 14 July 2010 /Accepted: 19 July 2010 /Published online: 4 September 2010

(C) The Author(s) 2010. This article is published with open access at Springerlink.com

\begin{abstract}
This overview addresses homocysteine and folate metabolism. Its functions and complexity are described, leading to explanations why disturbed homocysteine and folate metabolism is implicated in many different diseases, including congenital birth defects like congenital heart disease, cleft lip and palate, late pregnancy complications, different kinds of neurodegenerative and psychiatric diseases, osteoporosis and cancer. In addition, the inborn errors leading to hyperhomocysteinemia and homocystinuria are described. These extreme human hyperhomocysteinemia models provide knowledge about which part of the homocysteine and folate pathways are linked to which disease. For example, the very high risk for arterial and venous occlusive disease in patients with severe hyperhomocysteinemia irrespective of the location of the defect
\end{abstract}

Communicated by: Victor Kožich

Competing interest: None declared.

Presented at: the International Fulda-Symposium on Homocysteine, Folate and Cobalamin Disorders, Fulda, Germany November 11-13, 2009

H. J. Blom $(\bowtie)$

Metabolic Unit, Department of Clinical Chemistry

Institute for Cardiovascular Research (ICaR-VU),

VU University Medical Centre,

Amsterdam, The Netherlands

e-mail: h.blom@vumc.nl

\section{Y. Smulders}

Internal Medicine,

Institute for Cardiovascular Research (ICaR-VU),

VU University Medical Centre,

Amsterdam, The Netherlands in remethylation or transsulphuration indicates that homocysteine itself or one of its "direct" derivatives is considered toxic for the cardiovascular system. Finally, common diseases associated with elevated homocysteine are discussed with the focus on cardiovascular disease and neural tube defects.

\section{Homocysteine and folate metabolism}

Homocysteine is an amino acid not used in protein synthesis. Its role is to serve as an intermediate in methionine metabolism. Homocysteine itself is located at a branch-point of metabolic pathways: either it is irreversibly degraded via the transsulphuration pathway to cysteine or it is remethylated back to methionine (Fig. 1).

\section{Transmethylation}

Methionine adenosyltransferase (MAT) catalyzes the biosynthesis of S-adenosylmethionine (AdoMet) from methionine and ATP (Mudd and Cantoni 1958). MAT is encoded by two genes that display a tissue-specific expression pattern. $M A T 1 A$ encodes MAT I/III and is only expressed in adult liver, whereas MAT2A-expressing MAT II is present in almost all tissues (Kotb et al. 1997; Mato et al. 1997). AdoMet donates a methyl group to, for example, DNA, RNA, proteins and neurotransmitters. Over 100 different methyltransferases may exist. Each of these reactions produces S-adenosylhomocysteine (AdoHcy) which is a potent inhibitor of most methyltransferases. AdoHcy hydrolase (SAHH) hydrolyzes AdoHcy to adenosine and homocysteine. Because the equilibrium of SAHH favors AdoHcy 


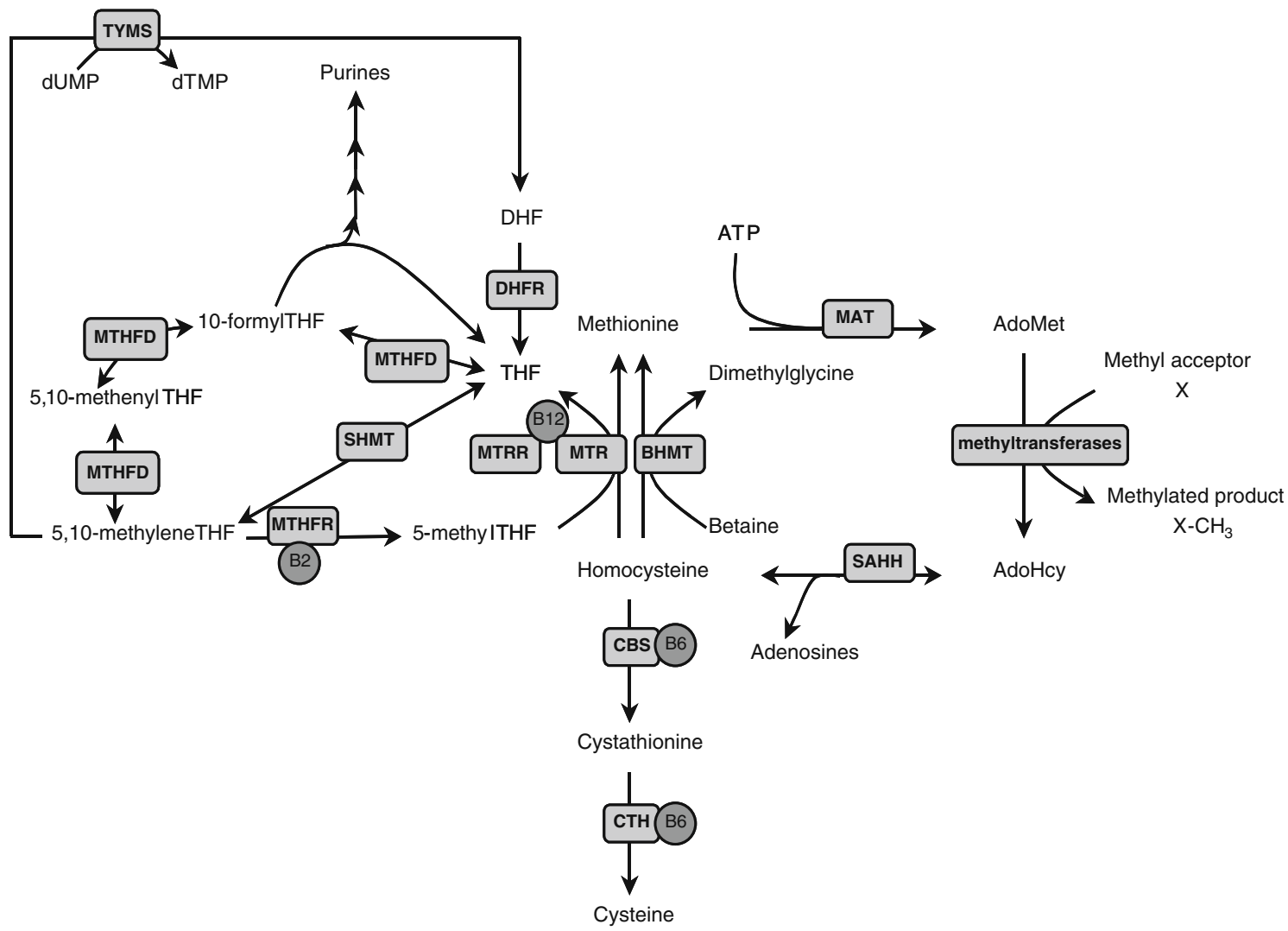

Fig. 1 Schematic representation of the folate cycles and homocysteine metabolism. AdoHcy S-adenosylhomocysteine, AdoMet Sadenosylmethionine, AICAR 5-aminoimidazole-4-carboxamide ribonucleotode, $S A H H$ S-adenosylhomocysteine hydrolase, ATP adenosine triphosphate, BHMT betaine-homocysteine methyltransferase, $C B S$ cystathionine $\beta$-synthase, $C T H$ cystathionine $\gamma$-lyase, $D H F$ dihydrofolate, DHFR dihydrofolate reductase, $d U M P$ deoxyuridine monophos-

formation, both homocysteine and adenosine need to be metabolized or transported out of the cell to prevent AdoHcy accumulation.

\section{Remethylation}

Homocysteine remethylation to methionine is catalyzed by the methionine synthase (MTR) enzyme (Li et al. 1996) and links the folate cycle with homocysteine metabolism (Fig. 1). MTR requires cobalamin (Cbl) as a cofactor, and the resulting complex, $\mathrm{Cbl}(\mathrm{I}) \mathrm{MTR}$, binds the methyl group of 5-methyTHF to form methylcbl(III)MTR. Upon transfer of the methyl group to homocysteine, Cbl(I) MTR is reformed, which can accept another methyl group from 5-methyltetrahydrofolate (5-methylTHF). $\operatorname{Cob}(\mathrm{I})$ alamin can also be oxidized to cob(II)alamin, which results in an inactive $\mathrm{Cbl}(\mathrm{II}) \mathrm{MTR}$ complex. Methionine synthase reductase (MTRR) reactivates the Cbl(II)MTR complex by reductive methylation, using AdoMet as a methyl donor (Leclerc et al. 1998). phate, dTMP deoxythymidine monophosphate, FAICAR formyl-AICAR, MAT methionine-adenosyltransferase, MTHFD methylenetetrahydrofolate dehydrogenase / methenyltetrahydrofolate cyclohydrolase / formyltetrahydrofolate synthetase, MTHFR methylenetetrahydrofolate reductase, MTR methionine synthase, MTRR methionine synthase reductase, SHMT serine-hydroxymethyltransferase, THF tetrahydrofolate, TYMS thymidylate synthase

Whereas the MTR enzyme is ubiquitously expressed, another homocysteine remethylation system, the betainehomocysteine methyltransferase (BHMT), is mainly expressed in the liver and kidneys (Sunden et al. 1997).

Transsulphuration

The homocysteine molecule is retained during remethylation and transmethylation reactions, but in the transsulphuration pathway homocysteine is irreversibly degraded to cysteine. Transsulphuration is facilitated by the action of two vitamin B6-dependent enzymes: cystathionine $\beta$ synthase (CBS) and cystathionine $\gamma$-lyase (CTH) (Fig. 1). CBS catalyzes the condensation of homocysteine and serine to cystathionine and CTH subsequently catalyzes the hydrolysis of cystathionine to cysteine and $\alpha$-ketobutyrate. Human CBS is expressed in liver, kidneys, muscle, brain and ovary and also during early embryogenesis in the neural and cardiac systems (Quere et al. 1999). Apart from its role in protein synthesis, cysteine is a precursor of 
glutathione, a strong antioxidant and an essential compound in detoxification of many xenobiotics.

\section{Regulation}

In most tissues, homocysteine is either remethylated via methionine synthase or exported out of the cell. The liver is the main organ of degradation of excess methionine and in maintaining homocysteine at adequate levels via an unique set of enzymes, including MAT I/III, CBS, CTH, BHMT, GNMT (glycine N-methyltransferase). In contrast to MAT II, MAT I/III has a high $\mathrm{Km}$. This produces that in the liver high methionine results in increased AdoMet. One main regulatory mechanism is that high levels of AdoMet inhibit MTHFR and activate CBS activity, respectively (Finkelstein 2007). Methionine excess thus results via the higher levels of AdoMet in homocysteine degradation via the transsulphuration pathway. Conversely, if methionine levels are low, for example during fasting, the low AdoMet levels will not activate CBS nor inhibit MTHFR, resulting in conservation of homocysteine via remethylation back to methionine.

\section{Folate cycles}

Intracellularly, 5-methylTHF functions as a methyl donor for homocysteine remethylation (Blom et al. 2006). The resulting THF can directly be converted into 5,10-methyleneTHF by the action of serine hydroxymethyltransferase (SHMT). SHMT is a vitamin B6-dependent enzyme that uses serine as a one-carbon donor. In humans, SHMT has a cytosolic as well as a mitochondrial isoform (Garrow et al. 1993). Conversion of THF into 5,10-methyleneTHF, via 10 -formylTHF and 5,10-methenylTHF, is catalyzed by the trifunctional enzyme methylenetetrahydrofolate dehydrogenase (MTHFD1) that has formyltetrahydrofolate synthetase, methenyltetrahydrofolate cyclohydrolase and methylenetetrahydrofolate dehydrogenase activities (Hum et al. 1988). The 10-formylTHF can donate one-carbon groups for purines biosynthesis, whereas 5,10-methylenetetrahydrofolate can be used as a cofactor for the conversion of dUMP into dTMP. The latter reaction is catalyzed by the thymidylate synthase (TYMS) enzyme and produces dihydrofolate (DHF), which requires subsequent reduction back to THF by the action of dihydrofolate reductase (DHFR). In addition to being a cosubstrate for dTMP synthesis, 5,10-methyleneTHF can also be reduced to 5-methylTHF by the riboflavin (vitamin B2)dependent enzyme methylenetetrahydrofolate reductase (MTHFR), which competes for 5,10-methyleneTHF with TYMS. The function of the MTHFR enzyme is of great importance for the regulation of available 5-methylTHF for homocysteine remethylation.
Folate uptake and transport

The water-soluble B-vitamin folate is an essential vitamin for humans and is obtained from the diet, especially from fruits and vegetables. According to chemical nomenclature, the difference between folate and folic acid is just one proton. However, the term folic acid is in general applied to the synthetic form of this B-vitamin, which is also the most stable form (Blom et al. 2006; Pitkin 2007). Dietary folates predominantly exist as polyglutamates, which have to be hydrolyzed to monoglutamates in order to be transported. The enzyme folylpoly- $\gamma$-glutamate carboxypeptidase (FGCP), that is anchored to the intestinal apical brush border and is encoded by the glutamate carboxypeptidase II (GCPII) gene, is responsible for this hydrolysis in the gut (Fig. 2) (Chandler et al. 1991). Monoglutamylated folates are subsequently absorbed in the duodenum and upper part of the jejunum by the high-affinity proton-coupled folate receptor PCFT1 (Qiu et al. 2006). 5-MethylTHF is the main form of folate circulating in the plasma and can be transported into the cell by means of carrier- or receptormediated transport. Folate receptor $\alpha(\mathrm{FR}-\alpha)$ is a glycosylphosphatidylinositol-linked glycoprotein with a high affinity for the monoglutamate 5-methylTHF and is expressed in a limited number of epithelial cells, predominantly in the proximal tubules of the kidney, the choroid plexus and the placenta (Wang et al. 1992; Barber et al. 1999; Kamen and Smith 2004). The other folate receptors, $\beta$ and $\gamma$, have a much lower affinity for 5methylTHF than FR- $\alpha$. Carrier-mediated transport occurs via the ubiquitously expressed reduced folate carrier (RFC) whose affinity for 5-methylTHF is also considerably lower than that of the FR- $\alpha$. The elucidation of the role of PCFT1 in cellular uptake and transport requires further research.

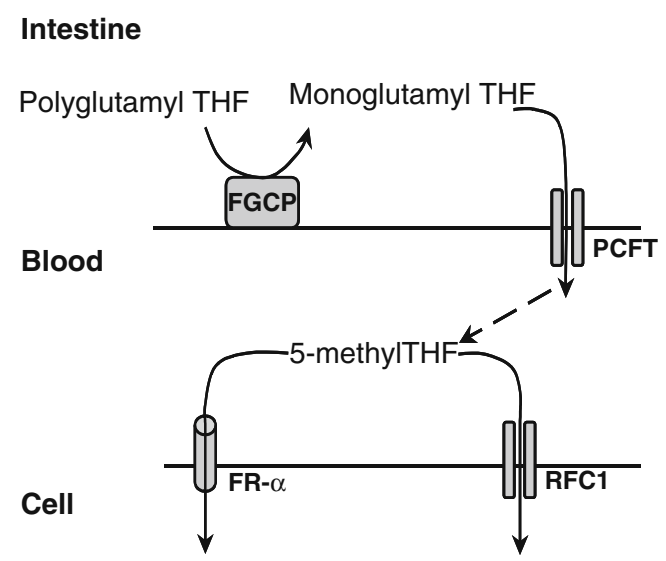

Fig. 2 Schematic overview of folate uptake and transport from the intestine, via the blood, into the cell. THF tetrahydrofolate, $F R-\alpha$ folate receptor alpha, $P C F T$ proton-coupled folate transporter, $R F C 1$ reduced folate carrier, $T H F$ tetrahydrofolate, $F G C P$ folylpoly- $\gamma$ glutamate carboxypeptidase 


\section{Inborn errors of homocysteine metabolism}

Severe hyperhomocysteinemia (total homocysteine $>50 \mathrm{uM}$ ) or homocystinuria is caused by defects in remethylation or transsulphuration. Disturbed remethylation can be caused by MTHFR as well as MTR deficiency due to mutations in their genes. MTR can also be dysfunctional due to defects in cobalamin metabolism. In MTR deficiency or dysfunction, 5-methylTHF cannot cycle through MTR resulting in 5methylTHF accumulation at the expense of the other folates, hampering the synthesis of purines and thymidine. In particular, rapidly dividing cells, such as bone marrow, will be affected and result in megaloblastic anemia and pancytopenia, which are also observed in folate deficiency.

MTHFR deficiency does not limit the availability of folates for purines and thymidine syntheses and therefore shows no abnormalities in blood cells.

Remethylation defects result in elevated homocysteine and decreased methionine. In the transsulfuration pathway, CBS deficiency also results in accumulation of homocysteine, but in contrast to remethylation defects, methionine is increased.

One typical characteristic of severe hyperhomocysteinemia is the wide array of different neurological presentations, ranging from schizophrenia and depression to severe mental retardation. Another common finding is arterial and venous occlusive disease in patients with severe hyperhomocysteinemia, irrespective of whether the defect is in remethylation or transsulphuration.

Interestingly, Yap et al (2001) showed a dramatic decrease in arterial and venous occlusive disease in CBSdeficient patients on homocysteine-lowering therapy.

\section{Disturbed homocysteine and folate metabolism as risk factor for vascular disease}

\section{Homocysteine and vascular disease}

In 1969, Kilmer McCully (McCully 1969) recognized the potentially harmful effects of homocysteine on the vascular wall. In two patients with severe hyperhomocysteinemia due to different inborn errors of homocysteine metabolism, he found similar vascular lesions. Because severely elevated plasma homocysteine concentration was the only common metabolic abnormality, he postulated the 'Homocysteine Theory'. He stated that homocysteine, or one of its derivatives, is toxic for the vascular wall (McCully 1969). In the years to follow, it became clear from both retrospective and prospective studies that also mildly elevated total homocysteine levels (of about $15 \mathrm{uM}$ ) are also independently associated with an increased risk for vascular diseases (Ueland et al. 2000). Whether or not this association is causal is still under debate. Large intervention trials have been performed and some are ongoing to address this issue. Recently, a meta-analysis of the Cochrane Database of Systematic Reviews was published (MartiCarvajal et al. 2009) including eight randomized controlled trials involving 24,210 participants. Homocysteinelowering therapy did not reduce the risk of non-fatal or fatal myocardial infarction, stroke, or death by any cause [RR (risk ratio) $1.03,95 \%$ CI 0.94 to 1.13 ; RR $0.89,95 \%$ CI 0.73 to 1.08 ; and RR $1.00,95 \%$ CI 0.92 to 1.09 , respectively]. The authors concluded there is no evidence to support the use of homocysteine-lowering therapy to prevent cardiovascular events. Their conclusion seems valid regarding myocardial infarction, given the small confidence interval. However, regarding stroke with a point estimate $\mathrm{RR}$ of 0.89 and a $95 \%$ confidence interval of 0.73 to 1.08 , one cannot exclude that homocysteine-lowering therapy may relate to a RR of about 0.80 for stroke, as observed in prospective case-control studies (Ueland et al. 2000; Wang et al. 2007).

\section{Homocysteine controversy}

Taken together, there appears to exist an apparent homocysteine controversy. We observed vascular disease in inborn errors of homocysteine metabolism, with a strong decrease in risk on homocysteine-lowering therapy. In addition, there is remarkable consistency in observational studies showing that elevated homocysteine is associated with increased cardiovascular disease risk. In apparent contrast, however, no risk reduction is found in homocysteine-lowering trials. These trials are mainly based on prescription of folic acid. As illustrated in Fig. 1, one of the main functions of folate metabolism is to provide one-carbon building blocks for the synthesis of purines and pyrimidine. In particular during rapid cell growth and stress, these building blocks are required for DNA and RNA synthesis. Thus, a side effect of folic acid administration may be the promotion of proliferation and inflammation, both of which are crucial processes taking place in the atherosclerotic plaque. Intake of high amounts of folic acid may thus be beneficial via the homocysteine lowering, but may also be harmful via destabilization the atherosclerotic plaque (see Smulders and Blom in this issue for further discussion).

\section{Disturbed homocysteine and folate metabolism as risk factor for neural tube defects}

Folic acid and neural tube defects

In 1976, Smithells et al. (1976) demonstrated decreased levels of several micronutrients, especially folate, in serum of women pregnant with a neural tube defect (NTD)- 
affected child during the first trimester. Subsequent small trials in women who had a NTD-affected pregnancy indicated that periconceptional supplementation with 0.4 $5 \mathrm{mg}$ of folic acid per day decreased the recurrence risk of NTDs about fourfold (Blom 2009). In 1991, the MRC Vitamin Study Research group conducted a large international double-blind placebo-controlled randomized trial in 1,817 women who had a previous NTD-affected pregnancy and demonstrated a $72 \%$ reduction in NTD recurrence risk for periconceptional folic acid supplementation (Vitamin Study Research Group 1991). The protective effect of periconceptional folic acid supplementation on NTD occurrence risk has mainly been demonstrated in retrospective case-control studies (Blom 2009). However, a large randomized controlled trial in Hungarian women showed that supplementation with multivitamins including $0.8 \mathrm{mg}$ folic acid reduced the first occurrence of NTDs by $93 \%$ (Czeizel and Dudas 1992).

The results of these studies led to the general advice for women planning a pregnancy to take $0.4 \mathrm{mg}$ of folic acid per day at least 1 month before conception and during the first trimester of pregnancy. Women who are at high risk because of a previous NTD-affected pregnancy are advised to take $4 \mathrm{mg}$ of folic acid daily in the periconceptional period (Pitkin 2007). In the years after the endorsement of these recommendations, the expected decline in NTD prevalence failed to occur. In 1998, the United States mandated folic acid fortification of enriched cereal grain products was followed by a decline of NTD rates of $20-30 \%$.

The observation that women who use folic acid periconceptional are at reduced risk for NTD-affected pregnancies is one of the most promising clues to the etiology of NTDs. In the search for mechanisms underlying the beneficial effect of folic acid in relation to NTDs, several mechanisms have been suggested. One mechanism playing a possible a role in NTD etiology is methylation, as discussed in more detail below.

\section{Methylation}

It is becoming more and more evident that, besides genetic variation and environmental factors, epigenetic mechanisms also play an important role in the etiology of complex diseases (Blom et al. 2006). Since NTDs are multifactorial in origin, epigenetic mechanisms may well be involved in their etiology. DNA methylation, which is reprogrammed during early embryogenesis (Reik et al. 2001), is part of the epigenetic code and is a chief regulator of gene expression (Dean et al. 2005), has been hypothesized to be associated with NTDs (Blom et al. 2006).

The methylenetetrahydrofolate reductase (MTHFR) enzyme has a unique function in that it regulates the availability of methyl groups for methylation reactions at the cost of purines and pyrimidine synthesis. The $677 \mathrm{C}>\mathrm{T}$ variant in the MTHFR gene renders a less active thermolabile MTHFR enzyme (Frosst et al. 1995), resulting in elevated levels of 10-formylTHF and a decreased availability of 5-methylTHF in red blood cells (Bagley and Selhub 1998). The observations that the MTHFR 677TT genotype decreases the availability of 5-methylTHF for methylation (Frosst et al. 1995), results in a reduction of global DNA methylation (Friso et al. 2002; Castro et al. 2004), and is a genetic risk factor for NTDs (van der Put et al. 1995; Blom et al. 2006) support the concept that DNA methylation plays an important role in NTD etiology. The observation that inactivation of the DNA methyltransferase DNMT3B disrupts de novo DNA methylation, and causes multiple developmental defects including NTDs in mice, further supports the importance of DNA methylation (Okano et al. 1999). In addition, culturing rat embryos on low methionine containing media-induced NTDs resulted in protein hypomethylation that was specific for those proteins located in the neural tube, indicating that protein methylation may also play a role in proper neural tube closure (Coelho and Klein 1990; Moephuli et al. 1997).

Using a chick embryo model, our group tested the hypothesis that methylation is crucial for proper neural tube closure (Afman et al. 2005). Inhibition of SAHH in our in vitro chick embryo model resulted in inhibition of methylation, as judged from the increased AdoHcy concentration and a decreased AdoMet/AdoHcy ratio, and a widening of the anterior neuropore (ANP). Comparable results were obtained by inhibition of MAT. Inhibition of MAT in cultured mouse embryos also decreased the AdoMet/AdoHcy ratio, and these embryos also presented with cranial closure defects (Dunlevy et al. 2006). The AdoMet/AdoHcy ratio in mothers of a NTDaffected child has been demonstrated to be decreased (Zhao et al. 2006). Only one study, however, has actually examined DNA methylation status in relation to NTDs (Al-Gazali et al. 2001). In that study, the AdoMet/AdoHcy ratio and DNA methylation were both decreased in a single case with trisomy 21 and spina bifida (Al-Gazali et al. 2001). Taken together, these studies have lead to the hypothesis that methylation is hampered in women that are pregnant with a NTD-affected child and that folic acid supplementation prevents NTDs by donating methyl groups, hereby improving methylation (Blom et al. 2006).

In the future, large-scale studies in which DNA methylation patterns of healthy newborns and those with a NTD will be characterized and compared using array-based approaches, which will shed more light on the association between an impaired DNA methylation and NTD etiology in humans and may identify new genes essential for neural tube closure. If an impaired DNA methylation is indeed 
associated with NTD etiology, the next step will be to examine whether folic acid supplementation of the mother can affect DNA methylation patterns in her child.

The ultimate goal of identifying genes that are under epigenetic control and involved in NTD etiology is the identification of molecular pathways that are essential for proper neural tube closure and can be manipulated and in this way prevent NTDs.

\section{Disturbed homocysteine and folate metabolism as risk factor for common diseases}

Given the essential roles of folate and homocysteine in cellular metabolism via donations of one-carbon units for purines, thymidine and methylation reactions, it is not surprising that impaired homocysteine and folate metabolism is associated not only with cardiovascular disease and neural tube defects but with a wide range of common diseases. These include congenital birth defects like congenital heart disease, cleft lip and palate, late pregnancy complications, different kinds of neurodegenerative and psychiatric diseases, osteoporosis and cancer. Whether or not folic acid or other means of homocysteine lowering forms of therapy will reduce the risk of these diseases remains a main focus of future research. Regarding congenital defects and late pregnancy complications, the design of a placebo-controlled intervention study is complicated because folic acid in a daily dosage of $0.4 \mathrm{mg}$ is advised to women planning a pregnancy.

Open Access This article is distributed under the terms of the Creative Commons Attribution Noncommercial License which permits any noncommercial use, distribution, and reproduction in any medium, provided the original author(s) and source are credited.

\section{References}

Afman LA, Blom HJ, Drittij MJ, Brouns MR, van Straaten HW (2005) Inhibition of transmethylation disturbs neurulation in chick embryos. Brain Res Dev Brain Res 158:59-65

Al-Gazali LI, Padmanabhan R, Melnyk S et al (2001) Abnormal folate metabolism and genetic polymorphism of the folate pathway in a child with Down syndrome and neural tube defect. Am J Med Genet 103:128-132

Bagley PJ, Selhub J (1998) A common mutation in the methylenetetrahydrofolate reductase gene is associated with an accumulation of formylated tetrahydrofolates in red blood cells. Proc Natl Acad Sci USA 95:13217-13220

Barber RC, Lammer EJ, Shaw GM, Greer KA, Finnell RH (1999) The role of folate transport and metabolism in neural tube defect risk. Mol Genet Metab 66:1-9

Blom HJ (2009) Folic acid, methylation and neural tube closure in humans. Birth Defects Res A Clin Mol Teratol 85:295-302
Blom HJ, Shaw GM, den Heijer M, Finnell RH (2006) Neural tube defects and folate: case far from closed. Nat Rev Neurosci 7:724731

Castro R, Rivera I, Ravasco P et al (2004) 5, 10-methylenetetrahydrofolate reductase (MTHFR) $677 \mathrm{C}->\mathrm{T}$ and $1298 \mathrm{~A} \rightarrow \mathrm{C}$ mutations are associated with DNA hypomethylation. J Med Genet 41:454458

Chandler CJ, Harrison DA, Buffington CA, Santiago NA, Halsted $\mathrm{CH}$ (1991) Functional specificity of jejunal brush-border pteroylpolyglutamate hydrolase in pig. Am J Physiol 260: G865-G872

Coelho CN, Klein NW (1990) Methionine and neural tube closure in cultured rat embryos: morphological and biochemical analyses. Teratology 42:437-451

Czeizel AE, Dudas I (1992) Prevention of the first occurrence of neural-tube defects by periconceptional vitamin supplementation. N Engl J Med 327:1832-1835

Dean W, Lucifero D, Santos F (2005) DNA methylation in mammalian development and disease. Birth Defects Res C Embryo Today 75:98-111

Dunlevy LP, Burren KA, Mills K, Chitty LS, Copp AJ, Greene ND (2006) Integrity of the methylation cycle is essential for mammalian neural tube closure. Birth Defects Res A Clin Mol Teratol 76:544-552

Finkelstein JD (2007) Metabolic regulatory properties of Sadenosylmethionine and S-adenosylhomocysteine. Clin Chem Lab Med 45:1694-1699

Friso S, Choi SW, Girelli D et al (2002) A common mutation in the 5, 10-methylenetetrahydrofolate reductase gene affects genomic DNA methylation through an interaction with folate status. Proc Natl Acad Sci USA 99:5606-5611

Frosst P, Blom HJ, Milos R et al (1995) A candidate genetic risk factor for vascular disease: a common mutation in methylenetetrahydrofolate reductase. Nat Genet 10:111-113

Garrow TA, Brenner AA, Whitehead VM et al (1993) Cloning of human cDNAs encoding mitochondrial and cytosolic serine hydroxymethyltransferases and chromosomal localization. J Biol Chem 268:11910-11916

Hum DW, Bell AW, Rozen R, MacKenzie RE (1988) Primary structure of a human trifunctional enzyme. Isolation of a cDNA encoding methylenetetrahydrofolate dehydrogenase-methenyltetrahydrofolate cyclohydrolase-formyltetrahydrofolate synthetase. J Biol Chem 263:15946-15950

Kamen BA, Smith AK (2004) A review of folate receptor alpha cycling and 5-methyltetrahydrofolate accumulation with an emphasis on cell models in vitro. Adv Drug Deliv Rev 56:1085-1097

Kotb M, Mudd SH, Mato JM et al (1997) Consensus nomenclature for the mammalian methionine adenosyltransferase genes and gene products. Trends Genet 13:51-52

Leclerc D, Wilson A, Dumas R et al (1998) Cloning and mapping of a cDNA for methionine synthase reductase, a flavoprotein defective in patients with homocystinuria. Proc Natl Acad Sci USA 95:30593064

Li YN, Gulati S, Baker PJ, Brody LC, Banerjee R, Kruger WD (1996) Cloning, mapping and RNA analysis of the human methionine synthase gene. Hum Mol Genet 5:1851-1858

Marti-Carvajal AJ, Sola I, Lathyris D, Salanti G (2009) Homocysteine lowering interventions for preventing cardiovascular events. Cochrane Database Syst Rev CD006612

Mato JM, Alvarez L, Ortiz P, Pajares MA (1997) S-adenosylmethionine synthesis: molecular mechanisms and clinical implications. Pharmacol Ther 73:265-280

McCully KS (1969) Vascular pathology of homocysteinemia: implications for the pathogenesis of arteriosclerosis. Am J Pathol 56:111128 
Moephuli SR, Klein NW, Baldwin MT, Krider HM (1997) Effects of methionine on the cytoplasmic distribution of actin and tubulin during neural tube closure in rat embryos. Proc Natl Acad Sci USA 94:543-548

Mudd SH, Cantoni GL (1958) Activation of methionine for transmethylation. III. The methionine-activating enzyme of Bakers' yeast. J Biol Chem 231:481-492

Okano M, Bell DW, Haber DA, Li E (1999) DNA methyltransferases Dnmt3a and Dnmt3b are essential for de novo methylation and mammalian development. Cell 99:247-257

Pitkin RM (2007) Folate and neural tube defects. Am J Clin Nutr $85: 285 \mathrm{~S}-288 \mathrm{~S}$

Qiu A, Jansen M, Sakaris A et al (2006) Identification of an intestinal folate transporter and the molecular basis for hereditary folate malabsorption. Cell 127:917-928

Quere I, Paul V, Rouillac C et al (1999) Spatial and temporal expression of the cystathionine beta-synthase gene during early human development. Biochem Biophys Res Commun 254:127-137

Reik W, Dean W, Walter J (2001) Epigenetic reprogramming in mammalian development. Science 293:1089-1093

Smithells RW, Sheppard S, Schorah CJ (1976) Vitamin dificiencies and neural tube defects. Arch Dis Child 51:944-950

Sunden SL, Renduchintala MS, Park EI, Miklasz SD, Garrow TA (1997) Betaine-homocysteine methyltransferase expression in porcine and human tissues and chromosomal localization of the human gene. Arch Biochem Biophys 345:171-174
Ueland PM, Refsum H, Beresford SA, Vollset SE (2000) The controversy over homocysteine and cardiovascular risk. Am J Clin Nutr 72:324-332

van der Put NM, Steegers-Theunissen RP, Frosst P et al (1995) Mutated methylenetetrahydrofolate reductase as a risk factor for spina bifida. Lancet 346:1070-1071

Vitamin Study Research Group (1991) Prevention of neural tube defects: results of the Medical Research Council Vitamin Study. MRC Vitamin Study Research Group. Lancet 338:131137

Wang X, Qin X, Demirtas H et al (2007) Efficacy of folic acid supplementation in stroke prevention: a meta-analysis. Lancet 369:1876-1882

Wang X, Shen F, Freisheim JH, Gentry LE, Ratnam M (1992) Differential stereospecificities and affinities of folate receptor isoforms for folate compounds and antifolates. Biochem Pharmacol 44:1898-1901

Yap S, Boers GH, Wilcken B et al (2001) Vascular outcome in patients with homocystinuria due to cystathionine betasynthase deficiency treated chronically: a multicenter observational study. Arterioscler Thromb Vasc Biol 21:20802085

Zhao W, Mosley BS, Cleves MA, Melnyk S, James SJ, Hobbs CA (2006) Neural tube defects and maternal biomarkers of folate, homocysteine, and glutathione metabolism. Birth Defects Res A Clin Mol Teratol 76:230-236 